\title{
Impact of Different Mobility Models on AODV Protocol in MANET with NS-2.35 and Bonnmotion-2.1a
}

\author{
B.A.S Roopa Devi ${ }^{1}$, Dr.J.V.R Murthy ${ }^{2}$, Dr.G.Narasimha ${ }^{3}$ \\ Associate Professor, Department of CSE, Pragati Engineering College, Kakinada, AP, India ${ }^{1}$ \\ Professor, Department of CSE, JNT University, Kakinada, AP, India ${ }^{2}$ \\ Associate Professor, Department of CSE, JNT University, Kakinada, AP, India ${ }^{3}$
}

\begin{abstract}
Mobile Adhoc Networks are self configuring,infrastructureless network (i.e) a network without any existing network infrstructure or centralized administrationn. To faclitate communication among the nodes in MANET, multiple hops are needed due to the limited range of wireless network interfaces. In order to exchange data among the nodes ,a routing protocol[1] is used to discover the routes. The goal of routing protocol is to efficiently construct a route between a pair of nodes with minimum routing overhead and bandwidth consumption. In this paper, the performance of the best mobility model with the use of AODV routing protocol is implemented. Link breaks and Average Link Changes are calculated for different mobility models like Random Walk,Gauss Markov,Manhattan using BONNMOTION 2.1a[5] by varying the Network Size,Simulation Time and Transmission Range in order to decide on the best mobility model. Further the correctness of the best mobility model is investigated by the assessment of performance paramaeters like packet delivery ratio (PDR), average end to end delay, and throughput. . NS-2.35[6] was used to produce the networks, services and situation personality. According to the simulation study,the best mobility model is manahattam mobility model with less number of link breaks and average link changes and also with the instigation of performance metrics.
\end{abstract}

Keywords: Mobile Ad Hoc Network, Mobility Model,Bonnmotion-2.1a,AODV,NS-2.35

\section{INTRODUCTION}

A mobile ad hoc network (MANET) represents a system of wireless mobile nodes that can freely and dynamically self - organize into arbitrary and temporary network topologies, allowing people and devices to seamlessly communicate without any preexisting Infrastructure. An ad hoc routing protocol is a convection or standard that controls how nodes come to agree on which way to route packets between computing devices in a MANET. Nodes do not have a priori knowledge of the topology of the network around them; they have to discover it. The basic idea is that a new node announces its presence and listens to broadcast announcements from its neighbors. The node learns about new near nodes and ways to reach them, and announces that it can also reach those nodes. As time goes on, each node knows about all the other nodes and one or more ways of how to reach them. The mobility models have a considerable effect on the performance of these routing protocols. Ad hoc networks are formed spontaneously and deployed during an emergency. The mobile nodes in the networks will freely move and communicate with each other. Due to high mobility an dynamic changing topology, a suitable mobility model for a particular network scenario. The change in mobility model affects the performance of routing protocols.

In this paper, the effect of different mobility models on AODV [3] (Reactive Protocol)'s performance is investigated in the first section. Performance comparison has also been conducted by varying node densities and number of hops. . cond section discusses about the On Demand Routing Protocol AODV.The Third Section describes the various mobility models .In Fourth Section discusses about the Bonnmotion Motion -2.1a and also includes the Link Breks and Average Link Changes for different Mobility Models.In Fifth Section,the performance paramaeters like Packet delievery Ratio,Throughput,EndtoEndDelay are calculated for different mobility models.Finally in the sixth section, we take into consideration the performance analysis in the fifth section and also compare with the parameters used for performance analysis result for simulations in section 4 and conclude the paper.

\section{ROUTING PROTOCOLS}

\section{A. (Ad-Hoc on Demand Distance Vector)}

It is an on-demand reactive protocol, where their major difference stems out from the fact that DSR uses source routing, in which a data packet carries the complete path to be traversed, however, in AODV, the source node and the intermediate nodes store the next-hop information corresponding to each how for data packet transmission, it uses distant vector routing algorithm This protocol also uses the messages RREQ (Route Request), RREP (Route Replies) and RERR (Route Errors) under UDP protocol and its method is almost the same as in DSR where the source node floods the RREQ in the network when a route for a destination is not available. The major difference between AODV and other on-demand routing protocols is 
that it uses a destination sequence number (DSN) to determine an up-to-date path to the destination. A node updates its path information only if the DSN of the current packet received is greater than the last DSN stored at the node. A RREQ carries the source identifier (SrcID), the destination identifier (DestID), the source sequence number $(\mathrm{SSN})$, the DSN, the broadcast identifier (BcastID), and the time to live (TTL) field. DSN indicates the freshness of the route that is accepted by the source. When an intermediate node receives a RREQ, it either forwards it or prepares a RREP if it has a valid route to the destination. The validity of a route at the intermediate node is determined by comparing the sequence number at the intermediate node with the destination sequence number in the RREQ. If a RREQ is received multiple times, which is indicated by the BcastID-SrcID pair, the duplicate copies are discarded. All intermediate nodes having valid routes to the destination, or the destination node itself, are allowed to send RREP packets to the source. Every intermediate node, while forwarding a RREQ, enters the previous node address and it's BcastID. A timer is used to delete this entry in case a RREP is not received before the timer expires. This helps in storing an active path at the intermediate node as AODV does not employ source routing of data packets. When a node receives a RREP, information about the previous node from which the packet was received is also stored in order to forward the data packet to this next node as the next hop toward the destination. All nodes active in the net transmit periodically hello messages (considered as special RREP messages). If one node does not receive hello from the neighbor means connection lost with them and they modify their routing table deleting that path. It also sends a RRER to the other neighbor nodes that used that path. It can be done easily because each node keeps a list of all the active nodes in each communication. The AODV routing protocol is considered for MANET with populations of tens to thousands of mobile nodes and can handle low, sensible and reasonably high mobility rates, as well as a variety of data travel levels. AODV has also been designed to decrease the dissemination of control traffic and eliminate overhead on data traffic in order to improve scalability and performance. Another main advantage of this protocol is that routes are recognized on demand and destination sequence numbers are used to and the latest route to the destination and the connection setup delay is lower.

One of the disadvantages of this protocol is that intermediate nodes can lead to in-consistent routes if the source sequence number is very old and the intermediate nodes have a higher but not the latest destination sequence number, thereby having stale entries. Also multiple RREP packets in response to a single RREQ packet can lead to heavy control overhead. Another disadvantage of AODV is that the periodic beaconing leads to unnecessary bandwidth consumption.

\section{III.MOBILITY MODELS}

The mobility models used in the simulation studies are presented, compared and explained in this section. A mobility model should attempt to mimic the movements of real mobile nodes. Changes in speed and direction must occur, and they must occur in reasonable time slots.

Different mobility models can be differentiated according to their measure of how two nodes are dependent in their motion. dependency and measure of how current velocity, magnitude and direction are related to previous velocity. Nodes having same velocity have high temporal dependency . Given below are the descriptions of mobility models

\section{A. Manhattan Mobility Model}

Manhattan model emulate the movement pattern of mobile nodes on streets. It can be useful in modeling movement in an urban area .The scenario is composed of a number of horizontal and vertical streets. Given below is example topography showing the movement of nodes for Manhattan Mobility Model with seventeen nodes? The map defines the roads along the nodes can move. Important Characteristics: Maps are used in this model too. However, the map composed of a number of horizontal and vertical streets. The mobile node is allowed to move along the grid of horizontal and vertical streets on the map. At an intersection of a horizontal and a vertical street, the mobile node can turn left, right or go straight with certain probability. Except the above difference, the inter-node and intra-node relationships involved in the Manhattan model are the same as in the freeway model. It too imposes geographic restrictions on node

\section{B. Random Walk Model}

The Random Walk model was originally proposed to emulate the unpredictable movement of particles in physics. It is also referred to as the Brownian Motion. Because some mobile nodes are believed to move in an unexpected way, Random Walk mobility model is proposed to mimic their movement behavior. The Random Walk model has similarities with the Random Waypoint model because the node movement has strong randomness in both models. We can think the Random Walk model as the specific Random Waypoint model with zero pause time.

However, in the Random Walk model, the nodes change their speed and direction at each time interval. For every new interval $t$, each node randomly and uniformly chooses its new direction $)(t \theta)$ tfrom $(0, \pi 2]$. In similar way, the new speed follows a uniform distribution or a Guassian distribution from $[0, \mathrm{~V}]$.. If the node moves according to the above rules and reaches the boundary of simulation field, the leaving node is bounced back to the simulation field. This effect is called border effect.

The Random Walk model is a memoryless mobility process where the information about the previous status is not used for the future decision. That is to say, the current velocity is independent with its previous velocity and the future velocity is also independent with its current velocity. 


\section{Gauss Markov Mobility Model}

The Gauss-Markov Mobility Model was first introduced by Liang and Haasand widely utilized.In this model, the velocity of mobile node is assumed to be correlated over time and modeled as a Gauss-Markov stochastic process. When the node is going to travel beyond the boundaries of the simulation field, the direction of movement is forced to flip 180 degree. This way, the nodes remain away from the boundary of simulation field.In the Gauss-Markov model, the temporal dependency plays a key role in determining the mobility behavior.

\section{CALCULATION OF LINK BREAKS, AVERAGE LINK CHANGES}

BONNMOTION-2.1a is the tool used to calculate Link Breaks and Average Link Changes in each of the mobility models. BONNMOTION 2.1a is the responsible to produce all movements' information in tcl according to the mobility model selected. When they are generated, the movement patterns present a brief period so we have to be alert to skip this first seconds since they do not present the properties of the mobility model wanted. For each simulation, the position and movements of the nodes are randomly selected as well as the traffic among them. BONNMOTION is the accountable for the random properties of the positions and movements of the nodes and for the traffic NS-2.35 random variables are used.The parameters link breaks and Average Link Changes are calculated by varying the node density and simulation time as well as the transmission range for different mobility models like Manhattan Mobilty Model, RandomWalk Mobility Model, Gauss Markov Mobility Model. The results are shown below in Fig a:for Manhattan Mobility Model.Fig b:for Gauss Markov Mobility Model.Fig c:for RandomWalk Mobility Model.

\begin{tabular}{|c|c|c|c|c|c|c|c|c|c|c|c|c|c|c|c|c|}
\hline & & \multicolumn{3}{|c|}{$=20$} & \multicolumn{3}{|c|}{$n=40$} & \multicolumn{3}{|c|}{$n=60$} & \multicolumn{3}{|c|}{$n=80$} & \multicolumn{3}{|c|}{$n=100$} \\
\hline & Th & 50 & & 10 & 5 & 75 & 100 & 5 & 75 & 100 & 50 & 75 & 100 & 50 & 75 & 100 \\
\hline \multirow[t]{2}{*}{$S T=$} & LB' & & & & & & & & & & & & & & & 14 \\
\hline & $c 5$ & 0.17 & 0.51 & 0.77 & 1.1 & 1.54 & 2.14 & 2.32 & 3.92 & 5.51 & 4,23 & 5.97 & 8.95 & 5.89 & 10.15 & 14.03 \\
\hline \multirow[t]{2}{*}{20} & & & & & & & & 10 & & & 38 & & & & & 33 \\
\hline & & 0.56 & 0.67 & 0.77 & 0.72 & 1.14 & 1.69 & 2.17 & 3.51 & 5.68 & 8.92 & 13.7 & 19.78 & 8.92 & 13.7 & . \\
\hline \multirow[t]{2}{*}{$T=180$} & & & 25 & 33 & 38 & 25 & 33 & 33 & 34 & 35 & 30 & 32 & 41 & 54 & 53 & 78 \\
\hline & s & 92 & 13.7 & 19.72 & 8.92 & 13.7 & 19.7 & 4.59 & 6.15 & 9.03 & 5.42 & 7.61 & 10.778 & 8.281 & 12.76 & 19.98 \\
\hline \multirow[t]{2}{*}{$S T=200$} & & & & & & & & 18 & & 38 & 52 & & 80 & & 64 & 100 \\
\hline & & 0.39 & 0.48 & 1.23 & 1.58 & 2.68 & 3.04 & 2.67 & 4.53 & 7.23 & 7.24 & 10.6 & 15.13 & 10.81 & 15.51 & 23.91 \\
\hline \multirow[t]{2}{*}{$S T=240$} & & & & & & & 24 & 43 & & 59 & 54 & 40 & 00 & & 87 & 25 \\
\hline & & 0.37 & 0.5 & 0.57 & 2.07 & 3.18 & 4.85 & 5.17 & 7.12 & 9.81 & 7.79 & 10.14 & 14.451 & 10.55 & 15.44 & 23.66 \\
\hline \multirow[t]{2}{*}{$\mathrm{ST}=3$} & & & & & & & 27 & 42 & & 71 & 66 & 75 & 118 & 104 & 106 & 144 \\
\hline & & 8 & & 0.92 & 2.44 & & & & & 10.55 & 58.29 & & 17.951 & 12.34 & 2 & \\
\hline
\end{tabular}

Fig a:Link Changes and Link Breaks for Manhattan Mobility Model.

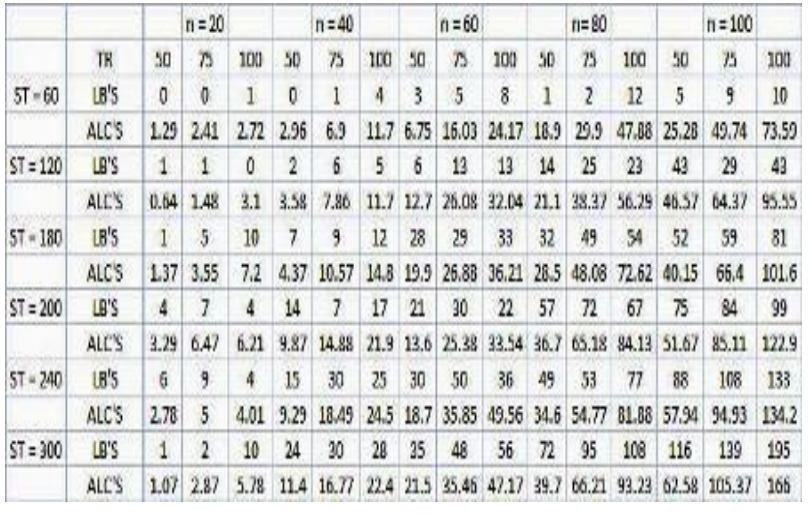

Fig b:Link Changes and Link Breaks for Gauss Markov Mobility Model.

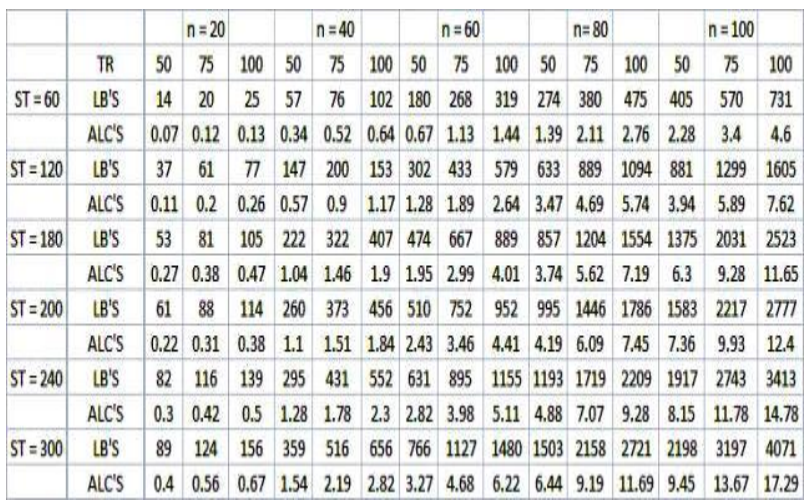

Fig c:Link Changes and Link Breaks for RandomWalk Mobility Model.

From the results, it is clear that the Manhattan Mobiltity Model exhibits less Link Breaks and Average link changes than the other mobility models.

\section{SIMULATION ENVIRONMENT}

\begin{tabular}{|c|c|}
\hline Parameter & Value \\
\hline Terrain Region & $1500 \mathrm{~m} \times 1500 \mathrm{~m}$ \\
\hline Routing Protocol & AODV \\
\hline Mobility model & Random Way Point \\
\hline Node Placement & Uniform Distribution \\
\hline Simulation Time & 200 sec \\
\hline Pause Time & 0 \\
\hline No. of nodes & $20,40,60,80,100$ \\
\hline Traffic & TCP \\
\hline Tool & Bonnmotion-2.1a \\
\hline Simulator & NS-2.35 \\
\hline
\end{tabular}




\section{PERFORMANCE METRICS AND RESULTS}

A. Performance Parameters The routing protocol's performance is measured with the following significant Quality of Services (QoS) metrics for routine measures:

1 Packet Delivery Ratio (PDR) Packet delivery ratio is an important metric as it describes the loss rate that will be seen by the transport protocols, which run on top of the network layer..It is the ratio of data packets delivered to the destination to those generated from the sources. It is calculated by dividing the number of packets received by destination through the number packet originated from the source. $\mathrm{PDF}=(\mathrm{Pr} / \mathrm{Ps}) * 100$ Where $\operatorname{Pr}$ is total Packet received \& Ps is the total Packet sent.

2 Throughput It is the average number of messages successfully delivered per unit time number of bits delivered per second . Throughput $=(\mathrm{kbits} / \mathrm{sec})$ Where $\mathrm{N}$ is the number of data sources.

3 Average End-to-End Delay It is defined as the time taken for a data packet to be transmitted across an Ad Hoc from source to destination. $\mathrm{D}=(\operatorname{Tr}-\mathrm{Ts})$, Where $\operatorname{Tr}$ is receive Time and Ts is sent Time.

\section{B. Result Analysis}

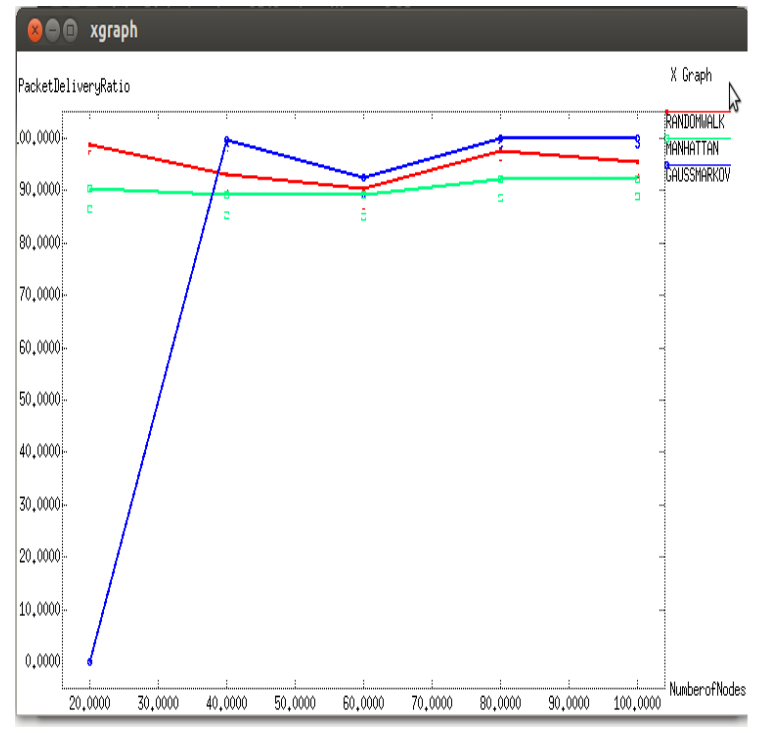

Fig. 1 Packet Delivery Ratio Vs Number of Nodes for Manhattan,RandomWalk,GaussMarkov Mobility Models

Packet Delivery Ratio remains consistent when varied with node density for manhattan mobility model as well as for RandomWalk Model.In the case of Gauss markov Model, there are no packets delievered at 20 node density which is inconsistent but performs well at node density of 100 nodes better than the other two mobility models.

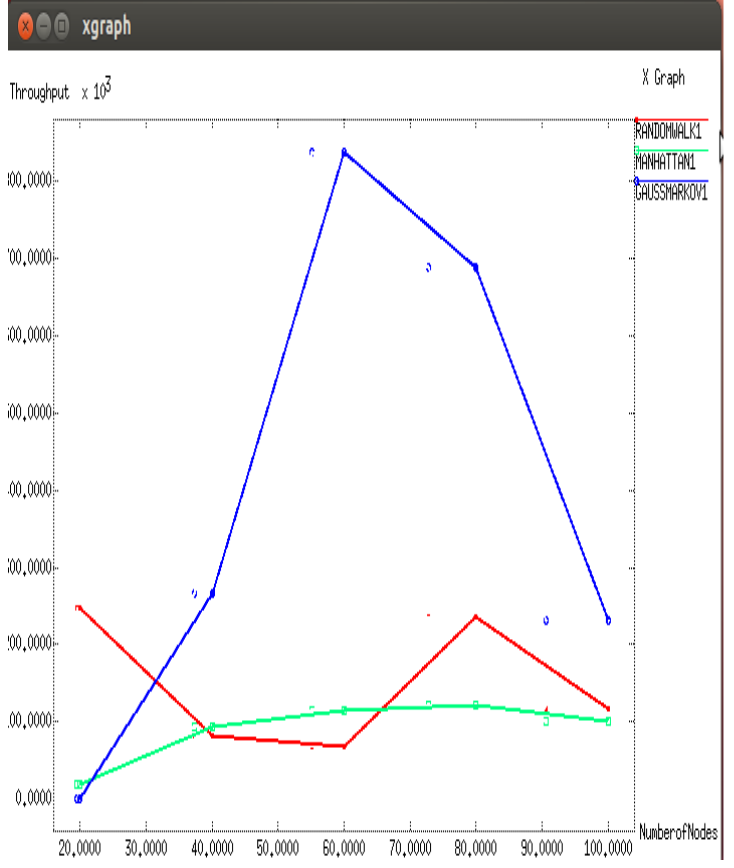

Fig. 2 Throughput Vs Number of Nodes for Manhattan,RandomWalk,GaussMarkov Mobility Models

Throughput for Randomwalk mobility model and Gauss Markov mobility model is not consistent with the node densitybut for manhattan model it is increases as the number of nodes increases and remains consistent throughout.

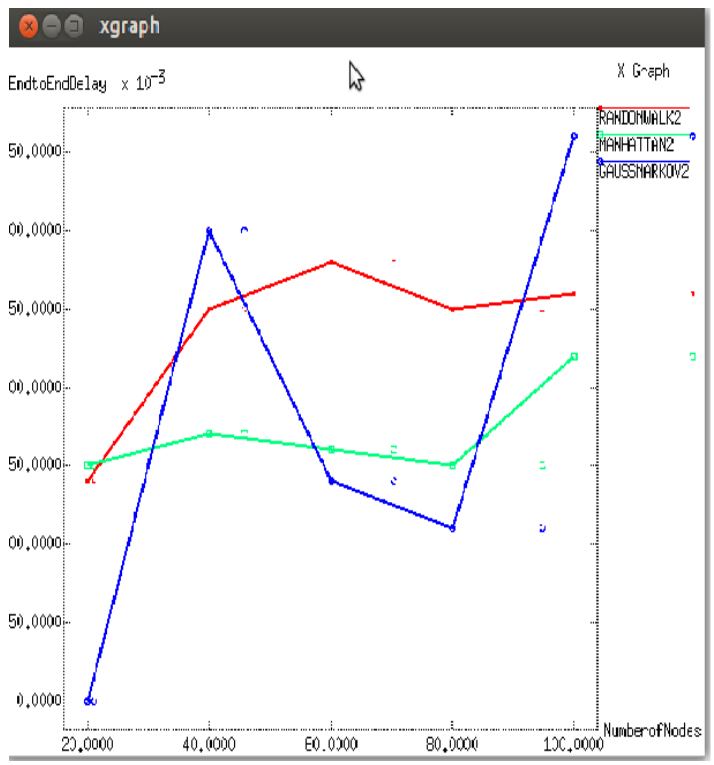

Fig. 3 EndtoEndDelay Vs Number of Nodes for Manhattan,RandomWalk,GaussMarkov Mobility Models

End to End Delay is minimum for manhattan model with respect to the number of nodes from 20 to 100,but for gauss markov increases rapidly at 40 nodes and 100 nodes .Also the Randomwalk mobility model is better than gauss markov mobility model but when compared to manhattan mobility model it doesnot perform efficiently. 


\section{VI .CONCLUSION}

In this paper, the impact of different mobility models on a ondemand routing protocol (AODV) has been investigated with respect to the Link breaks and Average Link Changes as well as the Qos metrics (a) PDR (b) Throughput (c) Average End to End delay. The performance of a routing protocol can vary significantly with different mobility models. The experimental results illustrate the good performance of different mobility models. Per experimental results, the performance of the protocol is greatly affected by the mobility model. According to the analysis ,Manhattan mobility model performs better when compared to other two mobility models (i.e) Randomwalk and GaussMarkov Mobility models in accordance with the parameters considered.

\section{ACKNOWLEDGMENT}

I would like to thank Dr.S Pallamsetty, for his valuable support in making of this paper. Further I extend my sincere thanks to my Guide Dr.J.V.R Murthy and CoGuide Dr.G.Narasimha for their guidance.

\section{REFERENCES}

[1] C. E. Perkins and P. Bhagwat, DSDV Routing over a Multi-hop Wireless Network of Mobile Computers. 2001.

[2] D. Johnson, Y. Hu, and D. Maltz, The dynamic source routing protocol (DSR) for mobile ad-hoc networks for IPv4", IETF, 2007.

[3] C. Perkins, E. Belding-Royer, and S. Das, lad-hoc on-demand distance vector (AODV) routing", IETF, 2003

[4] Network Simulator NS2 and Network Animator NAM. [Online].accessible: http://www.isi.edu/nsnam.

[5] M. Gerharz, C. de Waal, "Bonn Motion - a mobility scenario generation tool", University of Bonn, [Online]. Accessible: www.cs.uni-bonn.de/IV/BonnMotion/.

[6] T. Camp et al., "A Survey of Mobility Models for Ad Hoc Network Research", and Wireless Comm. \& Mobile Computing: Special issue on Mobile Ad Hoc Networking: Research, Trends and Applications, vol. 2, pp. 483-502, 2002.

[7] M. Umlauft and P. Reichl, Experiences with the ns-2 network simulator - explicitly setting seeds considered harmful", Proc. IEEE, 2007.

[8] Sham-ul-Arfeen et al, "Performance Evaluation of MANET Routing Protocols Using Scenario Based Mobility Models", Innovative Algorithmsand Techniques in Automation, IndustrialElectronics and Telecommunications, Springer, 2007. pp. 419-424

[9] Singh M, Singh D, Impact and Performance of Mobility Models in Wireless Adhoc Networks, Fourth Intern. Conference on Computer Sciences and Convergence Information Technology, 2009; 978-07695-3896.

[10]Sargolzaey H, Moghanjoughi AA, Khatun S. A review and comparison of reliable unicast routing protocols for mobile Ad-Hoc networks. Intern. J. Computer Sci. and Network Security. 2009: 9(1): 186-196.

[11] Kumar S, Sharma S C, Suman B. Mobility metrics based classification \& analysis of mobility model for tactical network. Intern. J. Of next-generation networks. 2010: 2(3): 39-51.

[12] Medina A, Gursun G, Basu P, Matta I. "On the Universal Generation of Mobility Models", in Proc. IEEE/ACM MASCOTS 2010, Miami Beach, FL, 2010.

[13] Medina A, Gursun G, Basu P, Matta I. " On the Universal Generation of Mobility Models", in Proc. IEEE/ACM MASCOTS 2010, Miami Beach, FL, 2010.

[14] Arvind Kumar Shukla, C K Jha and Deepak Sharma. Article: The Efficiency Analysis of Mobility Model using Routing Protocols.IJCA Proceedings on International Conference on Advances in Computer Applications 2012 ICACA(1):6-10, September 2012. Published by Foundation of Computer Science, New York, USA.

Copyright to IJARCCE
[15] Arvind Kumar Shukla, C K Jha and Deepak Sharma. Article: An Estimation of Routing Protocols in Mobility Models used for Ad Hoc Networks: Simulation Study. IJCA Proceedings on International Conference on Advances in Computer Application 2013 ICACA 2013:21-27, February 2013. Published by Foundation of Computer Science, New York, USA.

\section{BIOGRAPHIES}

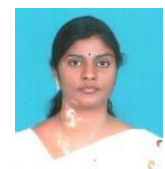

B.A.S Roopa Devi, has completed her B.Tech in Computer Science \& Engineering, J.N.T University Hyderabad, A.P. India in the year 2004, M.Tech in Software Engineering, J.N.T University Hyderabad, A.P. India in the year 2006, Currently pursuing her Ph.D in Computer Science and Engineering, J.N.T.University Kakinada.

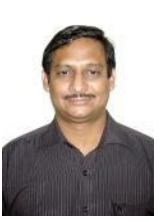

J.V.R Murthy, has completed his B. E in Electrical and Electronics Engineering, J.N.T University Hyderabad, A.P. India in the year1982, M.Tech in Computer Science and Data Processing, I.I.T Kharagpur, India in the year 1990, Ph.D in Computer Science and Engineering, J.N.T.University Hyderabad in the year 2005.

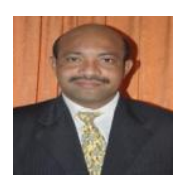

G.Narasimha has completed his B. E in Electronics and Communication Engineering, University College of Engineering, Osmania University Hyderabad, A.P. India in the year1996, M.Tech in Computer Science and Engineering, University College of Engineering, Osmania University Hyderabad, A.P. India in the year 1999, Ph.D in Computer Science and Engineering, University College of Engineering, Osmania University Hyderabad, A.P. India in the year 2009 